\title{
Relation between Inner Structural Dynamics and Ion Dynamics of Laser-Heated Nanoparticles
}

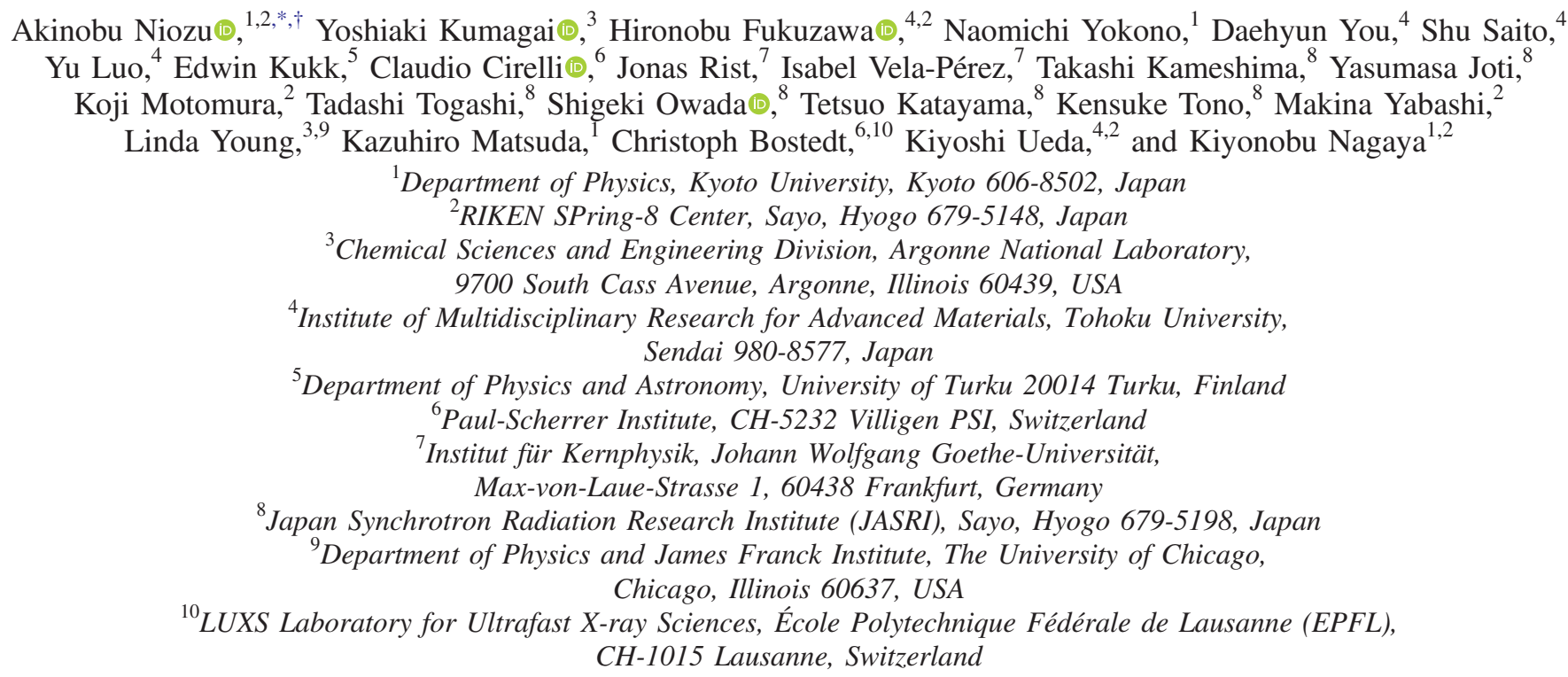

(Received 13 August 2020; revised 8 June 2021; accepted 30 June 2021; published 30 August 2021)

\begin{abstract}
When a nanoparticle is irradiated by an intense laser pulse, it turns into a nanoplasma, a transition that is accompanied by many interesting nonequilibrium dynamics. So far, most experiments on nanoplasmas use ion measurements, reflecting the outside dynamics in the nanoparticle. Recently, the direct observation of the ultrafast structural dynamics on the inside of the nanoparticle also became possible with the advent of x-ray free electron lasers (XFELs). Here, we report on combined measurements of structural dynamics and speeds of ions ejected from nanoplasmas produced by intense near-infrared laser irradiations, with the control of the initial plasma conditions accomplished by widely varying the laser intensity $\left(9 \times 10^{14} \mathrm{~W} / \mathrm{cm}^{2}\right.$ to $\left.3 \times 10^{16} \mathrm{~W} / \mathrm{cm}^{2}\right)$. The structural change of nanoplasmas is examined by timeresolved $x$-ray diffraction using an XFEL, while the kinetic energies of ejected ions are measured by an ion time-of-fight method under the same experimental conditions. We find that the timescale of crystalline disordering in nanoplasmas strongly depends on the laser intensity and scales with the inverse of the average speed of ions ejected from the nanoplasma. The observations support a recently suggested scenario for nanoplasma dynamics in the wide intensity range, in which crystalline disorder in nanoplasmas is caused by a rarefaction wave propagating at a speed comparable with the average ion speed from the surface toward the inner crystalline core. We demonstrate that the scenario is also applicable to nanoplasma dynamics in the hard x-ray regime. Our results connect the outside nanoplasma dynamics to the loss of structure inside the sample on the femtosecond timescale.
\end{abstract}

DOI: 10.1103/PhysRevX.11.031046

\footnotetext{
*Present Address: Graduate School of Advanced Science and Engineering, Hiroshima University, Higashi-hiroshima 7398526, Japan.

†niozu.akinobu.85s@kyoto-u.jp

Published by the American Physical Society under the terms of the Creative Commons Attribution 4.0 International license. Further distribution of this work must maintain attribution to the author(s) and the published article's title, journal citation, and DOI.
}

Subject Areas: Atomic and Molecular Physics, Plasma Physics

\section{INTRODUCTION}

The innovative technique of chirped pulse amplification of short laser pulses [1] has opened up new research areas in physics [2,3], chemistry [4-6], and laser engineering [7,8]. In recent years, novel applications of intense lasers have been brought about by the availability of short-wavelength free-electron lasers (FELs) $[9,10]$, which deliver extremely intense femtosecond pulses in the extreme ultraviolet and 
$\mathrm{x}$-ray regime. Such intense laser pulses, when focused to a narrow waist in the order of micrometers, can deposit an unprecedented amount of energy in a small volume of matter almost instantaneously. This extreme energy absorption in matter leads to the formation of a highly nonequilibrium plasma, resulting in an ultrafast structural change and ejection of charged fragments [7,8]. Characterizing and controlling the dynamics of laser-induced plasmas, typically on a femtosecond and nanometer scale, are essential for the applications. For instance, the laser-induced plasma dynamics is closely related to structure determination of biomolecules and transient species by ultrafast coherent diffraction imaging with x-ray FELs (XFELs) [11,12]. Here, the key concept is "diffraction before destruction" [13], in which the diffraction signal is recorded before significant structural changes take place in the sample due to radiation damage. The ultrashort but intense XFEL pulses are suitable for this time-critical technique, but for its proper implementation, understanding laser-induced plasma dynamics is of crucial importance in XFEL-based imaging experiments.

For the past few decades, nanometer-sized rare-gas clusters have commonly been used as a test bed to investigate fundamental dynamic processes in laserinduced plasma, called nanoplasma, since the produced nanoplasma has a uniform temperature profile and no energy dissipation to the surroundings. Regardless of the wavelength of the laser, the nanoplasma expands on a femtosecond to picosecond timescale, leading to ejection of highly charged energetic ions [14,15], fast electrons [16], and $\mathrm{x}$-ray/EUV photons $[17,18]$. To date, insight into the nanoplasma dynamics on femtosecond timescales and extreme energy scales has been predominantly obtained from secondary observables such as electron and ion kinetic energy releases, reflecting the nanoplasma expansion. However, the structural dynamics inside the particle during the solid-to-plasma transition and, here in particular, the loss of the atomic order remain unexplored.

The advent of XFELs has allowed novel single-shot single-particle imaging [19] and spectroscopy [20] approaches. Time-resolved imaging of near-infrared (NIR) laser-induced nanoplasmas allowed us, for the first time, to directly follow the initial steps of the nanoplasma expansion in space and time [21]. The data suggested a rapid softening of the nanoparticle surface, resulting in transient core-shell structures with shrinking solid-density cores and rapidly expanding outer shells. Wide-angle x-ray scattering (WAXS) with hard $x$ rays added first data on the structural dynamics inside a nanoplasma [22]. The experiments revealed the surprising results that the nanoplasma retain their inner crystalline order long (hundreds of femtoseconds) after the driving NIR laser pulse, in spite of the huge energy absorption in the electron system upon the excitation.

These pioneering experiments have provided qualitative information about the structural dynamics of laser-heated nanoparticles. From these observations, one can construct a hypothesis about the structural dynamics of a laserheated nanoplasma. After the laser excitation, the nanoparticle starts to expand by softening the surface density profile, and at the same time, a transition front propagates toward the interior, resulting in the crystalline disorder in the particle core. But key points remain open: Can we connect the inner structural dynamics and the outer nanoplasma expansion? How is the propagation of the transition front related to the ion dynamics or overall plasma dynamics? In theory $[23,24]$, the two aspects of the nanoplasma dynamics should be related via a parameter called plasma sound speed $c_{\mathrm{s}}$, defined as $\sqrt{Z k_{\mathrm{B}} T_{\mathrm{e}} / m_{\mathrm{i}}}$ ( $Z$ is the average charge, $k_{\mathrm{B}}$ is the Boltzmann constant, $T_{\mathrm{e}}$ is the electron temperature, and $m_{\mathrm{i}}$ is the atomic mass). The propagation of the transition front should correspond to a so-called rarefaction wave, which propagates with $c_{\mathrm{s}}$ from the surface towards the inner core $[23,24]$; meanwhile, the average speed of ejected ions is equivalent to $c_{\mathrm{s}}$ except for a factor of order unity [25].

In this work, we correlate the structural dynamics of nanoparticles irradiated by intense laser pulses and the kinetic energies of ions ejected from the produced nanoplasmas experimentally. The present experiment examines the nanoplasma dynamics induced by an intense NIR pulse. In this case, it can efficiently absorb energy through collective absorption processes, and the plasma parameters are widely controllable by the laser intensity [26]. First, we characterize the structural evolution of the nanoplasma with time-resolved WAXS on single particles. We control the plasma parameters ( $Z$ and $T_{\mathrm{e}}$ ) with the NIR laser parameters, resulting in intensity-dependent timescales of the plasma expansion and a corresponding change in kinetic energies of ejected ions. We overcome the common problem of focal volume averaging with a small crossing angle between the NIR pump laser and XFEL. With this geometry, and exploiting the angular information in the diffraction data, we can analyze the laser power-densitydependent processes and obtain a clear picture of the dynamics within the focal volume. Second, we measure the kinetic energies of the ions ejected from NIR-irradiated Xe clusters under the same conditions as the WAXS experiments. With these data sets, we identify a clear correlation between the delay-dependent diffraction signal and the kinetic energies of ejected ions: The timescale of the decay of the diffraction intensities scales with the inverse of the average speed of ejected ions. Furthermore, we demonstrate that the presented correlation may also apply to the dynamics of x-ray-induced nanoplasmas, which has been investigated by time-resolved WAXS in a previous work [27]. From the data, we can conclude that the crystalline disorder in nanoplasmas is universally caused by a transition front propagating towards the inner crystalline core at a speed comparable with the average speed of ejected ions. 


\section{EXPERIMENTS}

The experiments were performed at the experimental hutch EH2 [28] of beamline BL3 at the SPring-8 Angstrom Compact Free Electron Laser (SACLA) facility [29]. The setup is described in our previous publications [22,30]. SACLA generated $x$-ray pulses with a photon energy of $10.0 \mathrm{keV}$ at a repetition rate of $30 \mathrm{~Hz}$. The pulse duration of XFEL was estimated to be 10 fs [31]. The pulse energies were measured with a beam position monitor [28] located upstream of the beamline. The average pulse energy at the reaction point was $200 \mu \mathrm{J}$. The XFEL beam was focused with Be compound refractive lenses [32] to a focal size of $1.7 \mu \mathrm{m}$ (FWHM) The resulting peak intensity was $6 \times 10^{17} \mathrm{~W} / \mathrm{cm}^{2}$.

A Xe cluster beam was produced by an adiabatic expansion of Xe gas through a nozzle with a diameter of $200 \mu \mathrm{m}$ and a half angle of $4^{\circ}$. The stagnation pressure and temperature were 20 bar and $280 \mathrm{~K}$, respectively. The average cluster size was estimated to be $8 \times 10^{6}$ atoms/cluster (corresponding to approximately $50 \mathrm{~nm}$ in radius) according to Hagena's scaling law [33]. The cluster beam was introduced to the interaction region through two skimmers with diameters of $0.4 \mathrm{~mm}$ and $2.0 \mathrm{~mm}$.

NIR laser pulses with a wavelength of $\lambda=800 \mathrm{~nm}$ and a duration of 30 fs were generated by a Ti:sapphire laser system. The laser pulses were focused to a spot size of $30 \mu \mathrm{m}$ (FWHM) and overlapped with the XFEL pulses with a small crossing angle of approximately $1.5^{\circ}$, which was evaluated from the time-resolved diffraction data (see the Appendix A). The experimental geometry allowed us to analyze the power-density-dependent processes in the NIR focal volume as described in detail below. The spatial and temporal overlaps between XFEL and NIR laser pulses were ensured by measuring time-resolved ion spectra of the Xe clusters [34]. The pump-probe diffraction measurements were carried out at three NIR pulse energies-9 $\mathrm{mJ}$, $1 \mathrm{~mJ}$, and $0.2 \mathrm{~mJ}-$ and the resulting peak intensities were $3 \times 10^{16} \mathrm{~W} / \mathrm{cm}^{2}, 4 \times 10^{15} \mathrm{~W} / \mathrm{cm}^{2}$, and $9 \times 10^{14} \mathrm{~W} / \mathrm{cm}^{2}$, respectively. The shot-to-shot timing jitter between the XFEL and NIR pulses was compensated using the arrival timing monitor [35] with an accuracy of about $20 \mathrm{fs}$.

The scattered $\mathrm{x}$ rays were recorded on a shot-by-shot basis on a multiport CCD (MPCCD) sensor detector [36] located at $L=100 \mathrm{~mm}$ downstream of the interaction region. In the present setup, the detection area covers the momentum transfer range of 1.2-3.0 $\AA^{-1}$. Ion signals from the Xe clusters were recorded with an ion time-offlight (TOF) spectrometer [30].

\section{ANALYSIS}

\section{A. Hit finding and preanalysis}

In the time-resolved diffraction measurements, more than 200000 events were accumulated at each NIR peak intensity. Prior to the analysis, an averaged dark image was subtracted from the MPCCD images. Images with Bragg spots were filtered with a blog-finding algorithm [37]. The hit rate of the events, which is defined as the ratio of images containing Bragg spots to the total number of x-ray shots, was less than 5\%. For each Bragg spot in the single-shot diffraction images (specified with an index $i$ ), the radial position $r_{i}$ was evaluated.

\section{B. Structure of pristine Xe clusters}

Figure 1 shows the radial distribution of the detected Bragg spots from pristine Xe clusters. The diffraction pattern shows peaks at positions corresponding to known fcc reflections $\left(\{111\}_{\mathrm{fcc}}\right.$ and $\left.\{200\}_{\mathrm{fcc}}\right)$. In addition, a broad peak around $r \approx 38 \mathrm{~mm}$ is observed. The broad peak originates from the randomly stacked hexagonal close-packed (rhcp) structure in the clusters [38]. The diffraction patterns indicate that the Xe clusters contain both rhcp and fcc structures. In the following discussion, we focus on Bragg spots located on the sharp peak around $r \approx 36 \mathrm{~mm}$, which originate from both fcc and rhcp structures. Here, the intensities of the Bragg spots reflect the volume of the whole close-packed crystalline domains in the clusters according to the Debye-Scherrer formalism, in either the fcc or rhcp structure.

\section{Delay-dependent radial intensity distribution and focal volume effects}

For any strong-field-induced dynamics, the actual NIR intensity exposed on the sample is of utmost importance.

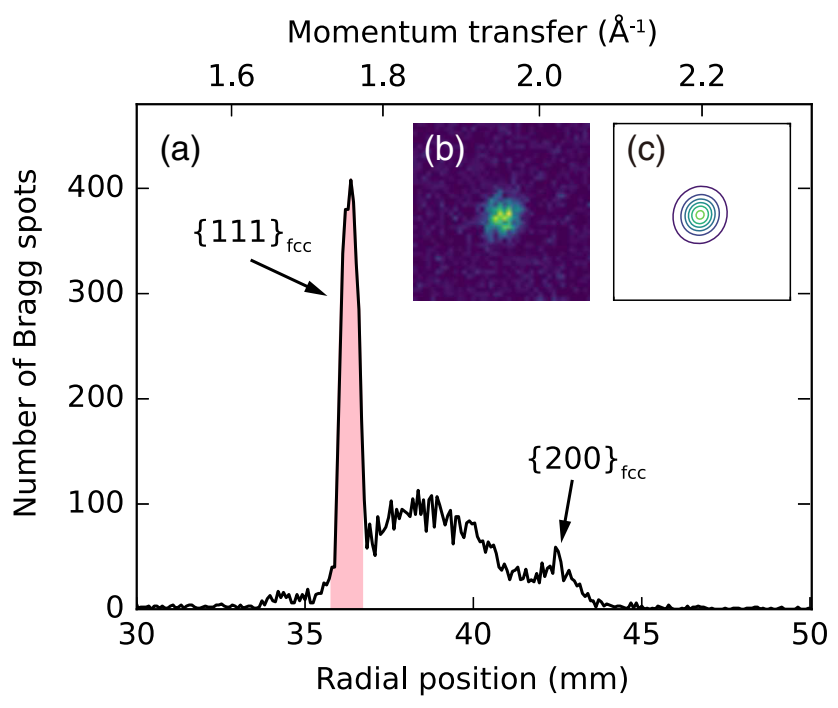

FIG. 1. (a) Radial distribution of the detected Bragg spots from pristine Xe clusters on the detector. The pattern exhibits peaks at positions corresponding to $\{111\}_{\text {fcc }}$ and $\{200\}_{\text {fcc }}$ reflections. The broad peak around $r \approx 38 \mathrm{~mm}$ originates from the rhcp structure [38]. In the subsequent analysis, we only focus on the Bragg spots located on the sharp peak at $r \approx 36 \mathrm{~mm}$ (filled with pink). (b) Representative example of a Bragg spot profile in a MPCCD image. (c) 2D Gaussian fit of panel (b). 
In our experiments, we were able to use the radial information in the x-ray diffraction data and our experimental geometry to gain a deeper insight into the NIRinduced processes in the nanoplasma. To do so, we first fit a two-dimensional (2D) intensity profile of each Bragg spot $i$ with a 2D Gaussian function. Then, the integrated intensity of the spot, $I_{i}$, was evaluated [cf. Figs. 1(b) and 1(c)]. We defined the unnormalized intensity $\widetilde{I}(r, t)$ as a function of the radial distance $r$ and the delay $t$ :

$$
\tilde{I}(r, t)=\sum_{i} I_{i} \delta\left(r-r_{i}\right) \delta\left(t-t_{i}\right),
$$

with $t_{i}$ being the delay of the event. The normalized intensity $I(r, t)$ was calculated as defined below:

$$
I(r, t)=\frac{\int_{r-\Delta r / 2}^{r+\Delta r / 2} \int_{t-\Delta t / 2}^{t+\Delta t / 2} \tilde{I}\left(r^{\prime}, t^{\prime}\right) d t^{\prime} d r^{\prime}}{\int_{t-\Delta t / 2}^{t+\Delta t / 2} N\left(t^{\prime}\right) d t^{\prime}},
$$

where $N(t)$ is the temporal distribution of the events, and $\Delta r$ and $\Delta t$ represent radial and temporal bin widths, respectively.

Figure 2(a) shows the delay-dependent radial intensity distribution $I(r, t)$ around the $\{111\}_{\text {fcc }}$ peak at the lowest NIR intensity: $I_{\text {peak }}^{\text {NIR }}=9 \times 10^{14} \mathrm{~W} / \mathrm{cm}^{2}$. We observe a decrease of the diffraction intensities around the center of the peak $(r \sim 36.3 \mathrm{~mm})$ within $1 \mathrm{ps}$ after the irradiation of NIR pulses. In contrast, Bragg spots at both ends of the peak survive even longer after the laser excitation.

The nonuniform delay dependence of the diffraction intensities can be attributed to the spatial intensity distribution of the NIR laser in the interaction region [Fig. 2(b)]. In the present experimental configuration, the NIR laser was overlapped with an XFEL beam with an intersection angle of $\phi \approx 1.5^{\circ}$, which was evaluated from the time-resolved diffraction data (see Appendix A). The width of the cluster beam was $\omega \approx 2 \mathrm{~mm}$ along the XFEL axis, which is smaller than the Rayleigh length of the XFEL. Along the XFEL beam axis, the NIR laser deviates from the XFEL beam axis by $(\omega \tan \phi) / 2 \approx 26 \mu \mathrm{m}$, which is comparable with the focal size of the NIR laser (30 $\mu \mathrm{m}$ in FWHM). Thus, the NIR intensity is not constant along the beam axis of XFEL, even though the Rayleigh length of the NIR laser is longer than the width of the cluster beam.

\section{Compensation of the NIR intensity distribution}

Equipped with this additional information from the experimental geometry and data, we can compensate, in the analysis, for the intensity variations in the focal volume (for details, see Appendix A). First, the spatial intensity distribution was computed by assuming a Gaussian beam profile. Figure 2(b) shows the calculated NIR intensity profile at $I_{\text {peak }}^{\text {NIR }}=9 \times 10^{14} \mathrm{~W} / \mathrm{cm}^{2}$. Then, the NIR intensity along the XFEL beam was associated with the radial profile using Bragg's law [cf. Fig. 2(d)]:

$$
\begin{gathered}
x=L-r / \tan 2 \theta_{111}, \\
\stackrel{\text { def }}{=} \Delta r / \tan 2 \theta_{111},
\end{gathered}
$$

where $x$ is the distance between the center of the XFEL focus and the position of the particle, and $\theta_{111}$ is the Bragg
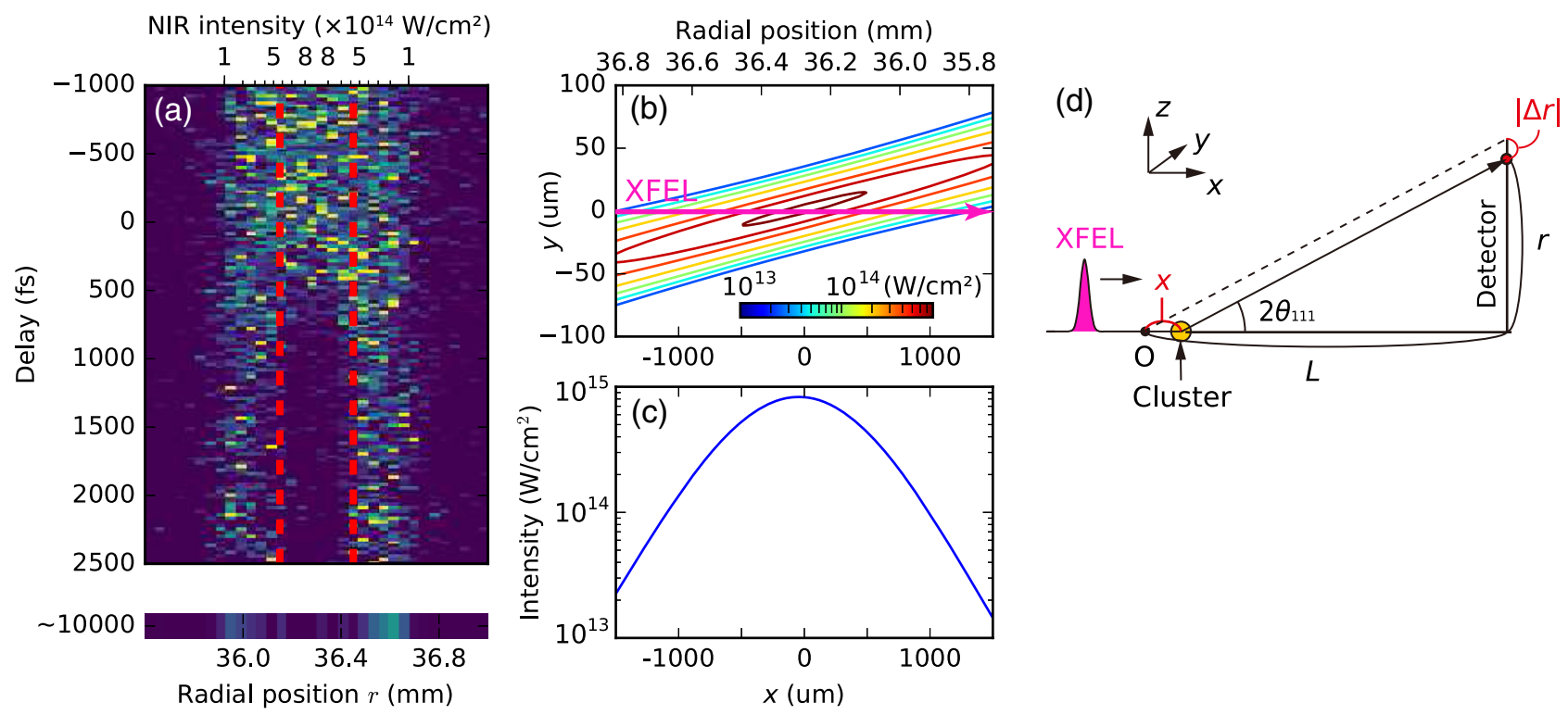

FIG. 2. (a) Delay-dependent radial profile of the diffraction intensities $I(r, t)$ around the $\{111\}_{\text {fcc }}$ peak at $I_{\text {peak }}^{\mathrm{NIR}}=9 \times 10^{14} \mathrm{~W} / \mathrm{cm}^{2}$. The upper axis indicates the estimated NIR intensity at the corresponding particle positions. (b) Top view of the spatial intensity distribution of the NIR laser in the interaction region. (c) NIR laser intensity distribution along the XFEL beam. (d) Schematic geometry of the diffraction measurements. 
angle for the $\{111\}_{\mathrm{fcc}}$ reflections. Here, we implicitly assumed that the Bragg angle $\theta_{111}$ is constant after irradiation with NIR pulses. This assumption is consistent with the present observations, where one does not see an evident collective shift of the radial positions of the Bragg spots. The upper axis of Fig. 2(a) indicates the calculated NIR intensity. The same procedure was applied to the three data sets recorded at three NIR peak intensities.

\section{E. Delay dependence of the diffraction intensities}

The NIR intensity distribution can now be taken into account in the analysis of the time-resolved diffraction data. We focused on diffraction intensities originating from near the focus of the NIR laser and computed the diffraction intensity $I(t)$ by integrating $\tilde{I}(r, t)$ over a radial range $\left[r_{\min }, r_{\max }\right]$ [indicated with red dashed lines in Fig. 2(a)]:

$$
I(t)=\frac{\int_{r_{\min }}^{r_{\max }} \int_{t-\Delta t / 2}^{t+\Delta t / 2} \tilde{I}\left(r^{\prime}, t^{\prime}\right) d t^{\prime} d r^{\prime}}{\int_{t-\Delta t / 2}^{t+\Delta t / 2} N\left(t^{\prime}\right) d t^{\prime}} .
$$

In addition, the NIR intensity in the range of interest was evaluated from the spatial intensity profile. The intensities were estimated to be $(2.2 \pm 0.6) \times 10^{16} \mathrm{~W} / \mathrm{cm}^{2}$, $(3.2 \pm 0.5) \times 10^{15} \mathrm{~W} / \mathrm{cm}^{2}$, and $(7.3 \pm 0.8) \times 10^{14} \mathrm{~W} / \mathrm{cm}^{2}$, respectively.

\section{RESULTS AND DISCUSSION}

\section{A. NIR intensity dependence}

The delay-dependent radial intensity distribution [Fig. 2(a)] provides key information about the NIR intensity dependence of the nanoplasma dynamics. The delaydependent profile exhibits a clear threshold behavior; diffraction intensities originating from the high NIR intensity region tend toward zero within 1 ps, while those from the low-intensity regions did not fade within $2.5 \mathrm{ps}$. The Bragg spots from the lower-intensity region survived even at $t \sim 10 \mathrm{ps}$. The threshold intensity for the disappearance of Bragg spots was roughly evaluated to be $5 \times 10^{14} \mathrm{~W} / \mathrm{cm}^{2}$. We should note that the threshold intensity is quite sensitive to the error in the intersection angle between the XFEL and the NIR laser. Interestingly, the threshold intensity coincides with the intensity where the onset of the energetic ion production was observed in ion measurements of Xe clusters $(N \sim 5300)$ [39]. This agreement implies a key relation between the properties of ejected ions and the structural dynamics: Serious structural damage is accompanied by energetic ion production.

Figure 3 shows the delay dependence of the Bragg diffraction intensities at the three NIR intensities. At all NIR intensities, the diffraction intensities start to decrease at $t=0$ and vanish within $1 \mathrm{ps}$. Most importantly, the data sets exhibit an evident NIR intensity dependence: The timescale of the decay is significantly longer at lower NIR

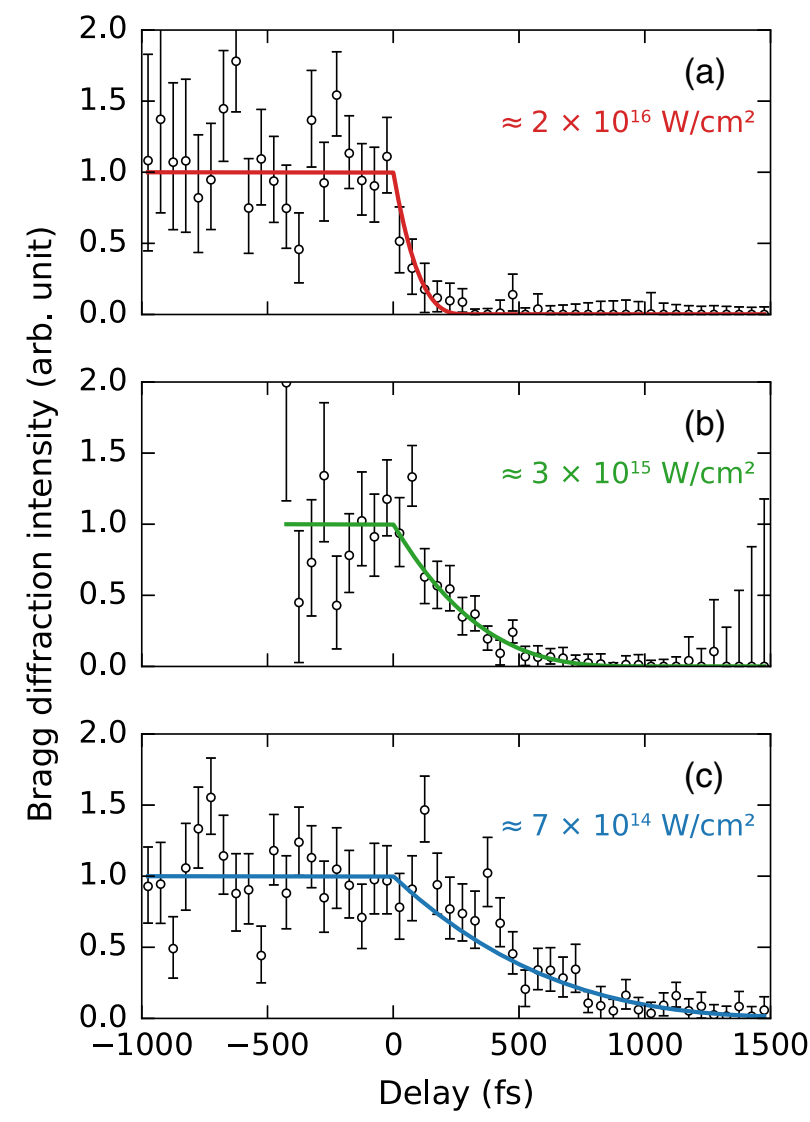

FIG. 3. Delay dependence of $\{111\}_{\text {fcc }}$ diffraction intensities of $\mathrm{Xe}$ clusters $I(t)$ at (a) $I^{\mathrm{NIR}}=(2.2 \pm 0.6) \times 10^{16} \mathrm{~W} / \mathrm{cm}^{2}$, (b) $I^{\mathrm{NIR}}=(3.2 \pm 0.5) \times 10^{15} \mathrm{~W} / \mathrm{cm}^{2}$, and (c) $I^{\mathrm{NIR}}=(7.3 \pm$ $0.8) \times 10^{14} \mathrm{~W} / \mathrm{cm}^{2}$. Solid curves show the fitting results with the nanoplasma model. For details of the model, see main text.

intensities. The decrease of the diffraction intensities indicates the loss of crystalline order in the Xe clusters. It should be emphasized that the crystalline order is retained for more than a few hundred femtoseconds. At the lowest NIR intensity, the diffraction intensities survived up to $1 \mathrm{ps}$. The timescale is much longer than the duration of the NIR laser pulses (30 fs in FWHM) and consistent with the timescale of nanoplasma expansion observed in previous studies [21,22]. The NIR intensity dependence of the structural dynamics can be interpreted as the difference in the initial parameters of the plasma; the higher laser intensity corresponds to higher $T_{\mathrm{e}}$ and $Z$.

\section{B. Structural dynamics inside the nanoplasma}

With the detailed power-density-dependent data at hand, we can discuss the structural evolution inside the nanoplasmas, in combination with the results from previous studies. Several experiments have shown that large, pristine, rare-gas clusters form a nearly spherical shape [40,41]. In addition, wide-angle $\mathrm{x}$-ray scattering experiments suggest that rare-gas clusters mostly consist of single, 
close-packed crystals [38]. After NIR excitation, clusters start to expand from the surface, resulting in the emergence of transient core-shell structures with solid-density cores [21]. The surface softening matches well with the theories of plasma expansion [23], in which surface ions are accelerated outwards by hydrodynamic forces, and at the same time, a rarefaction wave propagates inwards at a constant speed. The surface softening has also been verified in a microscopic simulation of NIR-irradiated hydrogen clusters [42]. The nanoplasma core retains its crystallinity until the transition front propagates to the core [22].

Based on the previous and current work, we employ a simple model, namely, the surface "melting" model, to explain the temporal evolution of the diffraction intensity. In the model, a spherical crystal starts to expand from the surface, and a transition front propagates towards the inner core at a constant speed $v_{\text {prop }}$ :

$$
R_{\text {core }}= \begin{cases}R_{0} & (t<0) \\ R_{0}-v_{\text {prop }} t & \left(0 \leq t<R_{0} / v_{\text {prop }}\right) \\ 0 & \left(t \geq R_{0} / v_{\text {prop }}\right),\end{cases}
$$

where $R_{\text {core }}$ is the core radius and $R_{0}$ is the average radius of the pristine clusters $(=50 \mathrm{~nm})$. In this model, we assume that the core completely retains its crystalline structure; i.e., the contribution of crystalline disorder in the core (core disorder) to the decrease of diffraction intensity is neglected. This assumption is consistent with the present observation, in which we observe no abrupt decrease of the diffraction intensities at $t \sim 0$. The diffraction intensity is assumed to be proportional to the volume of the crystalline core:

$$
I(t)=I_{0}\left(\frac{R_{\text {core }}}{R_{0}}\right)^{3}+B,
$$

where $I_{0}$ is the initial diffraction intensity and $B$ is the baseline. The baseline $B$ may originate from the diffraction signal from clusters located out of the NIR laser focus since the rhcp structure gives rise to a broad peak in the radial intensity distribution. The delay dependence of the diffraction intensities is fitted with Eqs. (6) and (7) (the solid curves in Fig. 3), and the propagation speed $v_{\text {prop }}$ is evaluated for each laser intensity. The fitted propagation speeds $v_{\text {prop }}$ are plotted against the NIR intensity in Fig. 4. The propagation speed varies by about 1 order of magnitude over the measured NIR intensity range $\left(7 \times 10^{14}-2 \times 10^{16} \mathrm{~W} / \mathrm{cm}^{2}\right)$.

\section{Connection of the inside dynamics to the outside dynamics}

To get a quantitative picture of the NIR intensity dependence of the structural dynamics, we deduce central plasma parameters from ion TOF spectra of NIR-irradiated Xe clusters. With this approach, we are then able to connect
NIR peak intensity $\left(\mathrm{W} / \mathrm{cm}^{2}\right)$

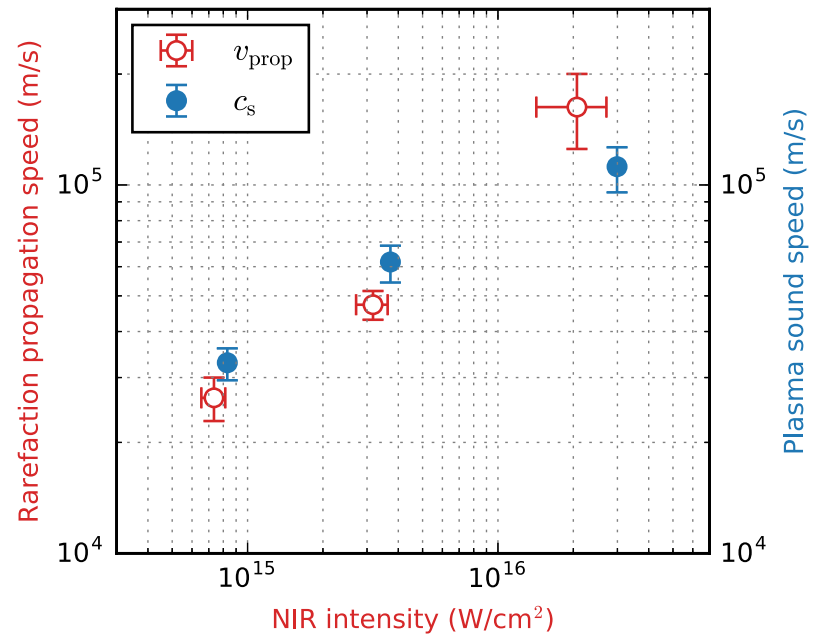

FIG. 4. Red points: propagation speeds $v_{\text {prop }}$ of the crystals deduced from the delay dependence of the diffraction intensities plotted against NIR intensity. The NIR intensities are evaluated by considering the spatial intensity profiles (see the main text and Appendix A). The vertical error bars indicate uncertainties originating from the statistical errors in the diffraction intensities. Blue points: plasma sound speeds deduced from the ion TOF spectra of the NIR-irradiated Xe clusters plotted against NIR peak intensity. The vertical error bars indicate uncertainties corresponding to the standard deviation of the ion kinetic energies in the fitting range.

the propagation of crystalline disorder inside the particle to the large body of work about particle explosion dynamics from ion spectra. Figure 5 shows the ion TOF spectra recorded at the same three NIR peak intensities as in the time-resolved diffraction measurements. At higher NIR intensities, highly charged ions with high kinetic energies are observed, indicating high ionization and strong heating in the clusters. Considering the spatial intensity distribution in the interaction region [20,43], we focus on ion signals in the highest charge states (corresponding to approximately $5 \%$ of the total ion yield), which can be assumed to originate from the focus of the NIR laser. The kinetic energies of the ions at the highest charge states are simulated and fitted to the experimental data (for details, see Appendix B). The average kinetic energies of ions $E$ at the highest charge states are evaluated to be $20 \pm 5 \mathrm{keV}$, $6.0 \pm 1.4 \mathrm{keV}$, and $1.7 \pm 0.3 \mathrm{keV}$, respectively.

In hydrodynamic expansion, the average ion kinetic energy $E$ approximates $\beta Z k_{\mathrm{B}} T_{\mathrm{e}}$ [25], where $\beta$ is a factor of order unity. We adopt a numerical factor $\beta=2 \times(3)^{-1 / 2}$, which corresponds to the expansion of quasineutral collisionless plasma in spherical geometry $[25,44]$. The plasma sound speed $c_{s}$ is estimated from the average ion kinetic energy via $E=2 \times(3)^{-1 / 2} m_{\mathrm{i}} c_{\mathrm{s}}^{2}$. The plasma sound speed $c_{s}$ is related to the ion speed $v_{\mathrm{i}}=\sqrt{2 E / m_{i}}$ via $v_{\mathrm{i}}=2 \times(3)^{-1 / 4} c_{s} \approx 1.52 c_{s}$. 

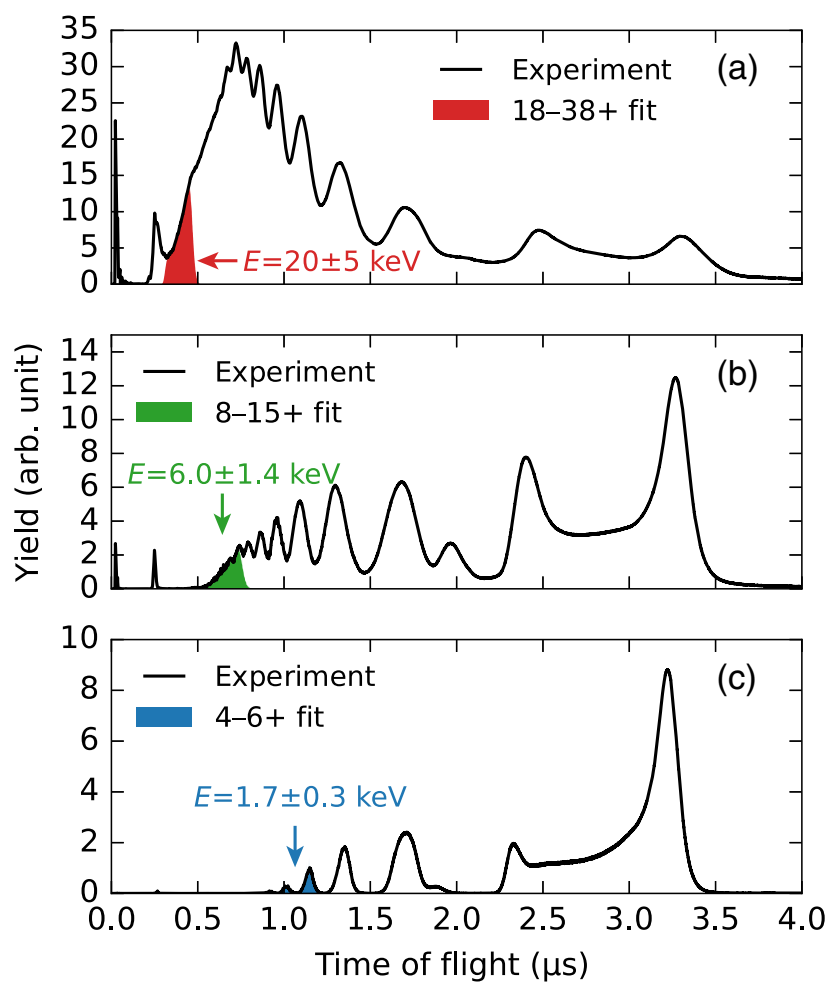

FIG. 5. Ion TOF spectra of NIR-irradiated Xe clusters at (a) $I_{\text {peak }}^{\mathrm{NIR}}=3 \times 10^{16} \mathrm{~W} / \mathrm{cm}^{2}$, (b) $I_{\text {peak }}^{\mathrm{NIR}}=4 \times 10^{15} \mathrm{~W} / \mathrm{cm}^{2}$, and (c) $I_{\text {peak }}^{\mathrm{NIR}}=9 \times 10^{14} \mathrm{~W} / \mathrm{cm}^{2}$. Ion TOFs at the highest charge states are simulated with SIMION and fitted with the experimental data (for details, see Appendix B).

In Fig. 4, the estimated plasma sound speeds are plotted against the NIR peak intensities (blue points). The plasma sound speeds surprisingly agree with the propagation speeds $v_{\text {prop }}$ deduced from the temporal evolution of the diffraction intensities. The agreement strongly supports the scenario of the surface softening $[21,23,45]$ in the wide range of excitation conditions, in which disordering propagates from the surface toward the crystalline core at the plasma sound speed $c_{s}$. Note that the uncertainty in the average cluster radius [46] causes a systematic vertical shift of the propagation speeds in the log-log plot. This relative shift between the propagation speeds and the plasma sound speed is offset by the choice of $\beta$ [25] used to derive the plasma sound speeds. Hence, though the present data do not necessarily guarantee the accuracy of $\beta$, the results are consistent with the scenario of the surface softening. The slight deviation between the propagation speed and the plasma sound speed in the highest intensity region could be due to the above-presented uncertainties or the core disorder, which means the breakdown of the assumption that the crystalline order is retained in the nanoplasma core.

\section{Nanoplasma dynamics in the hard x-ray regime}

Above, we have seen the relation between the inner structural dynamics and the outer ion dynamics of nanoplasmas produced in intense NIR laser fields. The following question now arises: Is the relation universally applicable to laser-induced nanoplasma dynamics in different excitation mechanisms, e.g., extreme ultraviolet and $\mathrm{x}$-ray regimes? There are several studies supporting the scenario of surface softening in x-ray-induced nanoplasmas; it has been observed in a time-resolved imaging of hard x-ray-induced nanoplasmas [47] and has also been verified in a numerical simulation of the explosion dynamics of a biomolecule irradiated by an intense, hard x-ray pulse [45]. Hence, it is reasonable to assume that nanoplasma expansion starts from the surface of the particle in a similar manner to the NIR regime.

As for the atomic order in hard $\mathrm{x}$-ray-induced nanoplasmas, Ferguson et al. [27] have reported on a timeresolved WAXS experiment of Xe nanoparticles (with a radius of $35 \mathrm{~nm}$ ) irradiated by intense, hard x-ray pulses. In their results, the diffraction intensity exhibits an approximately $40 \%$ decrease at $80 \mathrm{fs}$ after the irradiation of the $\mathrm{x}$-ray pulse. In their interpretation, the decrease in the diffraction intensity was solely attributed to the local atomic displacement. However, another explanation would be possible in light of present experimental results: The decrease in the diffraction intensity is explained by the reduction of the crystalline volume. Let us investigate the consistency between the experimental results [27] and the model proposed here. To evaluate the propagation speed $v_{\text {prop }}$ of the transition front towards the inner core, the delay-dependent diffraction intensity was fitted with the functions, Eqs. (6) and (7) (see Appendix C). The fitting procedure gives a value of $(6.2 \pm 0.6) \times 10^{4} \mathrm{~m} / \mathrm{s}$ for $v_{\text {prop}}$. From an ion TOF measurement similar to the present experiments, the average kinetic energy of ions ejected from the XFEL-induced nanoplasma is $10 \mathrm{keV}$ [27], giving a plasma sound speed $c_{s}=8 \times 10^{4} \mathrm{~m} / \mathrm{s}$ in the plasma model with $\beta=2 / \sqrt{3}$. The plasma sound speed is in good agreement with the propagation speed $v_{\text {prop. }}$. Though the available data are not sufficient to fully validate our model, the agreement suggests the possibility of a more universal relation between the inner structural dynamics and the ion properties, regardless of the excitation mechanism specific to the laser wavelength.

\section{E. Validity of the nanoplasma model}

As we have seen, the experimental results are well explained by the theory of collisionless plasma expansion. As a final point, we want to discuss the plasma description and verify our approach from several perspectives [39]. First, the quasineutrality is a good approximation, in the present case, of large clusters with heavy atoms; most of the electrons released from ions are confined in the emerging positive cluster potential. The second requirement for the plasma description is that the cluster size needs to be larger than the Debye length. Using the plasma parameters 
deduced from the ion spectra, the Debye length is calculated to be on the order of a few angstroms, which is much smaller than the cluster radius. Finally, the duration of the laser pulse has to be much shorter than the timescale of the plasma expansion. In the highest intensity case, the timescale of the plasma expansion is a few hundred femtoseconds, which is still one order longer than the duration of the laser pulse.

In the case of quasineutral plasma expansion, ion motion in the core is greatly suppressed by the charge neutrality due to the effective screening with the trapped electrons [45]. Meanwhile, there is room for consideration on whether the disorder in the crystalline core is negligible or not. Even if the ion motion in the core is negligible, the excitation can affect the atomic form factor, which can result in a decline of the diffraction intensity. In the present work, the experimental results are well explained by the simple model, which does not account for the core disorder. However, the unidentified core disorder might modify the above structural model. For a complete understanding of the disordering process, numerical simulations, including the excitation processes and the microscopic dynamics of plasmas, will be helpful.

\section{CONCLUSION}

In conclusion, we investigated the ultrafast structural evolution of nanoplasmas and correlated the inside rarefaction wave propagation to the outside ion speeds through the plasma parameters. Ultrafast, time-resolved diffraction shows that the timescale for the loss of crystalline order strongly depends on the NIR laser pump intensity, with higher intensities leading to faster propagation speeds of the transition front. Ion TOF spectra recorded at the same experimental conditions allowed us to determine common plasma parameters. Our analysis shows that the propagation speed of the transition front inside the nanoplasma is similar, though in the opposite direction, to the ejected ion speed multiplied by a factor of order unity, in good agreement with theory.

As some theories suggest [23], the relation might be general for matter under intense laser irradiation, irrespective of the pump laser intensity and wavelength, and thereby potentially applicable to a wide area of matter in extreme conditions generated in intense laser fields. Based on our findings in the present study, one can correlate a large body of literature on the outside dynamics of laserinduced plasma (e.g., high-energy ion production [14,25] and laser ablation mass spectroscopy) to that of the inner structural dynamics, which is directly related to the evolution of the laser-induced nanoplasma in the femtosecond temporal and nanometer spatial resolutions (e.g., laser fabrication and machining $[7,8])$.

Another important application is the issue of radiation damage caused by intense $\mathrm{x}$-ray irradiation. The connection between the loss of structure inside the sample and ion speeds of the hydrodynamic sample expansion allows simple experimental feedback on the ultrafast damage mechanisms. Thus, our findings may offer a requirement for single-shot diffraction measurements of nanometersized targets, which is of central importance for future applications of XFELs.

\section{ACKNOWLEDGMENTS}

The experiments were performed at SACLA with the approval of JASRI and the program review committee (No. 2018B8063). This study was supported by the Japan Society for the Promotion of Science (JSPS) KAKENHI Grants No. JP15K17487 and No. JP16K05016, and by JSPS Grant-in-Aid for JSPS Fellows No. JP19J14969. A. N. acknowledges support from the Research Program for Next Generation Young Scientists of "Dynamic Alliance for Open Innovation Bridging Human, Environment and Materials" in "Network Joint Research Center for Materials and Devices." K. N. acknowledges support by the Research Program of "Dynamic Alliance for Open Innovation Bridging Human, Environment and Materials" in "Network Joint Research Center for Materials and Devices." Y. K. and L. Y. acknowledge support from the U.S. Department of Energy, Office of Science, Basic Energy Science, Chemical Sciences, Geosciences and Biosciences Division under Contract No. DE-AC02-06CH11357. E. K. acknowledges support from the Academy of Finland. C. B. acknowledges the Swiss National Science Foundation National Center of Competence in Research-Molecular Ultrafast Science and Technology NCCR-MUST

\section{APPENDIX A: COMPENSATION OF THE NIR INTENSITY VARIATION}

The spatial profile of NIR intensity was computed by assuming a Gaussian beam with a horizontal rotation angle of $\phi$ :

$$
\begin{aligned}
& I^{\mathrm{NIR}}\left(r^{\prime}, z^{\prime}\right)=I_{\text {peak }}^{\mathrm{NIR}}\left(\frac{\omega_{0}}{\omega\left(z^{\prime}\right)}\right)^{2} \exp \left(-\frac{2 r^{\prime 2}}{\omega^{2}\left(z^{\prime}\right)}\right), \\
& \omega\left(z^{\prime}\right)=\omega_{0} \sqrt{1+\left(z^{\prime} / z_{\mathrm{R}}^{\prime}\right)^{2}} \\
&\left(\begin{array}{c}
r^{\prime} \\
z^{\prime}
\end{array}\right)=\left(\begin{array}{cc}
\cos \phi & -\sin \phi \\
\sin \phi & \cos \phi
\end{array}\right)\left(\begin{array}{l}
x-x_{\mathrm{c}} \\
y-y_{\mathrm{c}}
\end{array}\right),
\end{aligned}
$$

where $\omega_{0}=\mathrm{FWHM} / \sqrt{2 \ln 2}$ is the waist radius, $z_{\mathrm{R}}^{\prime}=$ $\pi \omega_{0}^{2} / \lambda$ is the Rayleigh range, and $\left(x_{\mathrm{c}}, y_{\mathrm{c}}\right)$ are the coordinates for the NIR beam waist. Below, we describe the procedure to determine the parameters: $x_{\mathrm{c}}, y_{\mathrm{c}}$, and $\phi$. First, we neglect the shift of the beam waist along the XFEL beam axis and assume $x_{\mathrm{c}}=0$ because the shift has just a minor effect on the intensity profile. Second, we determine $y_{\mathrm{c}}$ from the delay-dependent radial intensity profile so that 

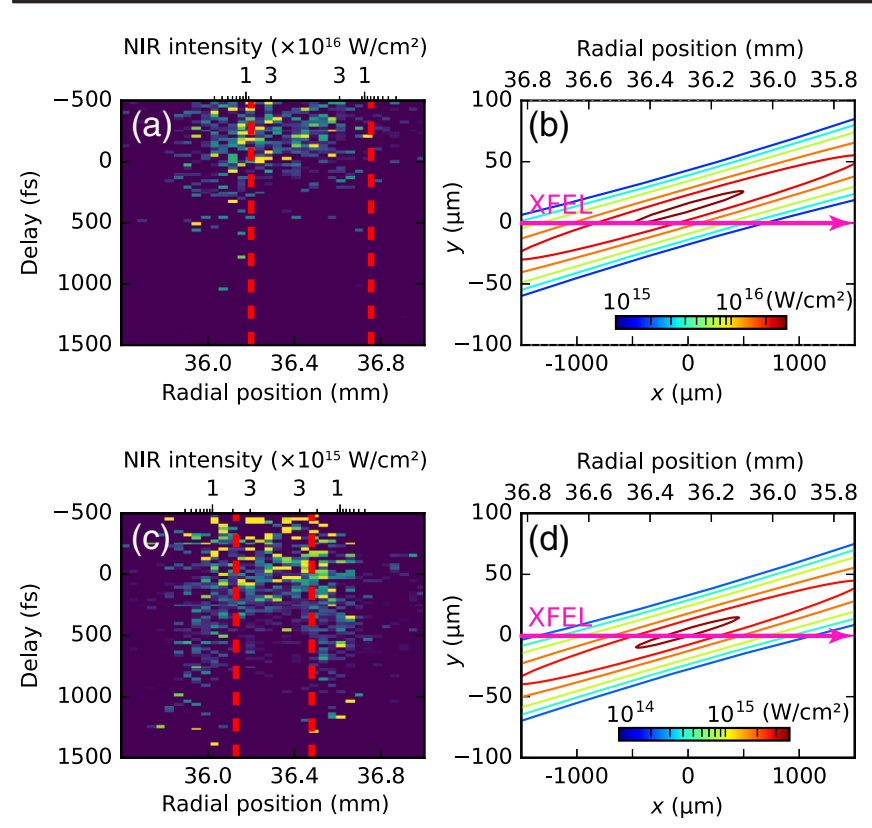

FIG. 6. (a,c) Delay-dependent radial profile of the diffraction intensities around the $\{111\}_{\text {fcc }}$ peak at (a) $I_{\text {peak }}^{\text {NIR }}=3 \times 10^{16} \mathrm{~W} / \mathrm{cm}^{2}$ and (c) $I_{\text {peak }}^{\mathrm{NIR}}=4 \times 10^{15} \mathrm{~W} / \mathrm{cm}^{2}$. The upper axis indicates the estimated NIR intensity at the interaction region. (b,d) Top view of the spatial intensity distribution of the NIR laser at (b) $I_{\text {peak }}^{\mathrm{NIR}}=3 \times 10^{16} \mathrm{~W} / \mathrm{cm}^{2}$ and (d) $I_{\text {peak }}^{\mathrm{NIR}}=4 \times 10^{15} \mathrm{~W} / \mathrm{cm}^{2}$.

the maximum of the NIR intensity corresponds to the dip in the profile. Note that $y_{\mathrm{c}}$ is determined independently for the three data sets since the measurements are performed at different mirror positions. Finally, we determine $\phi$ so that the calculated NIR intensity can consistently explain the delay-dependent radial intensity profiles at three NIR peak intensities [Figs. 6(a) and 6(c)]. We tried several values for $\phi$, with $\phi=1.5^{\circ}$ consistently giving the best results of the three data sets. This value is in reasonable agreement with the experimental geometry. The calculated intensity profiles are shown in Figs. 6(b) and 6(d).

The spatial laser profile is associated with the radial position of the Bragg spots via Eq. (4). Here, we neglect the focal size of XFEL and assume $y=0$ because it is one order smaller than that of the NIR laser. Substituting the coordinates $(x, y)=\left(L-r / \tan 2 \theta_{111}, 0\right)$ into Eq. (A3) and using Eqs. (A1) and (A2), the NIR intensity is calculated as a function of the radial positions of the Bragg spots. The upper axes of Figs. 6(a) and 6(c) indicate the calculated NIR intensity.

\section{APPENDIX B: EVALUATION OF KINETIC ENERGY FROM ION TOF SPECTRA}

To determine the ion kinetic energy from the ion TOF spectra, we use the ion optics simulation program SIMION. The determination of ion kinetic energy is not straightforward because of the problem of peak separation. As an example, here we present the analysis procedure for the ion
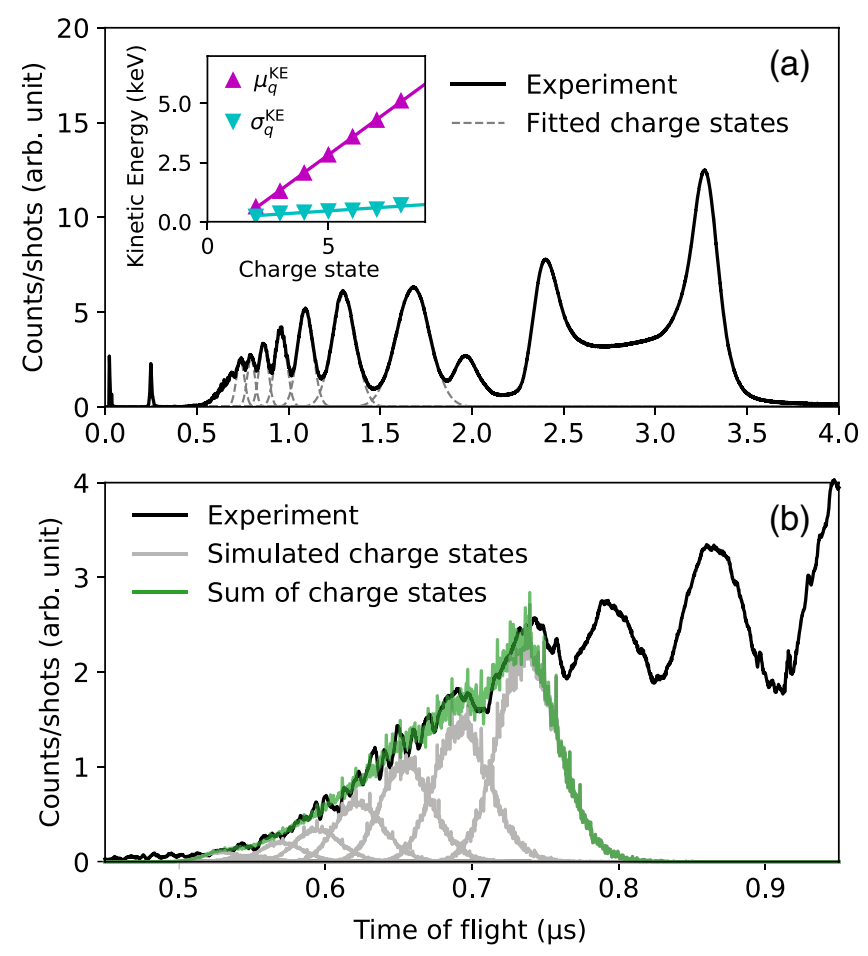

FIG. 7. (a) Ion TOF spectrum of NIR-irradiated Xe clusters at $I_{\text {peak }}^{\text {NIR }}=4 \times 10^{15} \mathrm{~W} / \mathrm{cm}^{2}$. The spectrum was fitted with a sum of Gaussian functions (dotted lines). Inset: correlation between the charge state and the ion kinetic energy. (b) Experimental spectrum fitted with the sum of the charge-state-resolved spectra simulated with an ion optics simulation software.

spectra at $I_{\text {peak }}^{\mathrm{NIR}}=4 \times 10^{15} \mathrm{~W} / \mathrm{cm}^{2}$. Similar procedures were applied to the other two spectra.

First, we focus on the ion signals at lower charge states ( $2 \leq q \leq 8)$, where the peaks are well separated. The peaks are fitted with the sum of Gaussian functions:

$$
y^{\mathrm{fit}}(T)=\sum_{2 \leq q \leq 8} a_{q} \exp \left[-\frac{\left(T-\mu_{q}\right)^{2}}{2 \sigma_{q}^{2}}\right]
$$

where $T$ is the time of flight, and $a_{q}, \mu_{q}$, and $\sigma_{q}$ represent the height, mean, and sigma of the Gaussian function for charge state $q$. The fitted curves are shown in Fig. 7(a). Note that we ignore the back peaks originating from ions initially ejected in the direction opposite to the detector. With the fitted parameters $\mu_{q}$ and $\sigma_{q}$, we evaluate the mean and the standard deviation of the ion kinetic energies: $\mu_{q}^{\mathrm{KE}}$ and $\sigma_{q}^{\mathrm{KE}}$. For this purpose, we employ time-to-energy conversion functions $E(q ; T)$ for ions toward the detectors [48], which were obtained by simulating TOFs of ions with various initial kinetic energies. Using the conversion functions $E(q ; T), \mu_{q}^{\mathrm{KE}}$ and $\sigma_{q}^{\mathrm{KE}}$ are calculated via

$$
\mu_{q}^{\mathrm{KE}}=E\left(q ; \mu_{q}\right)
$$




$$
\sigma_{q}^{\mathrm{KE}}=\left(E\left(q ; \mu_{q}-\sigma_{q}\right)-E\left(q ; \mu_{q}+\sigma_{q}\right)\right) / 2 .
$$

The obtained $\mu_{q}^{\mathrm{KE}}$ and $\sigma_{q}^{\mathrm{KE}}$ are plotted in the inset of Fig. 7(a). One can see a linear dependence of $\mu_{q}^{\mathrm{KE}}$ and $\sigma_{q}^{\mathrm{KE}}$ on the charge state $q$. The linear dependence of the kinetic energy has been interpreted as a signature of hydrodynamic expansion $[15,49]$.

By extrapolating the linear dependence, we estimate $\mu_{q}^{\mathrm{KE}}$ and $\sigma_{q}^{\mathrm{KE}}$ for higher charge states $(9 \leq q \leq 15)$. Then, charge-state-resolved TOF spectra $y^{\mathrm{sim}}\left(q, \mu_{q}^{\mathrm{KE}}, \sigma_{q}^{\mathrm{KE}} ; T\right)$ are simulated with the extrapolated and fitted parameters $\mu_{q}^{\mathrm{KE}}$ and $\sigma_{q}^{\mathrm{KE}}$. In the simulation, ion TOFs of 100000 initially directed throughout the forward hemisphere are simulated. Finally, the experimental ion spectra at the highest charge states $(q \geq 8)$ are fitted with sum of the simulated charge-state-resolved spectra:

$$
y^{\mathrm{fit}}(T)=\sum_{8 \leq q \leq 15} a_{q}^{\prime} \cdot y^{\mathrm{sim}}\left(q, \mu_{q}^{\mathrm{KE}}, \sigma_{q}^{\mathrm{KE}} ; T\right),
$$

with $a_{q}^{\prime}$ the fitting coefficient for the spectrum of charge state $q$. Figure 7(b) shows the fitted spectra together with the charge-state-resolved spectra. The average kinetic energy of ions at the highest charge states is calculated via

$$
E=\frac{\sum_{8 \leq q \leq 15} a_{q}^{\prime} \cdot \mu_{q}^{\mathrm{KE}}}{\sum_{8 \leq q \leq 15} a_{q}^{\prime}}
$$

One might notice that the experimental ion spectrum is slightly more structured than the fitting results, possibly because of the overestimation of $\sigma_{q}^{\mathrm{KE}}$. However, the error in $\sigma_{q}^{\mathrm{KE}}$ hardly influences the estimation of the average ion

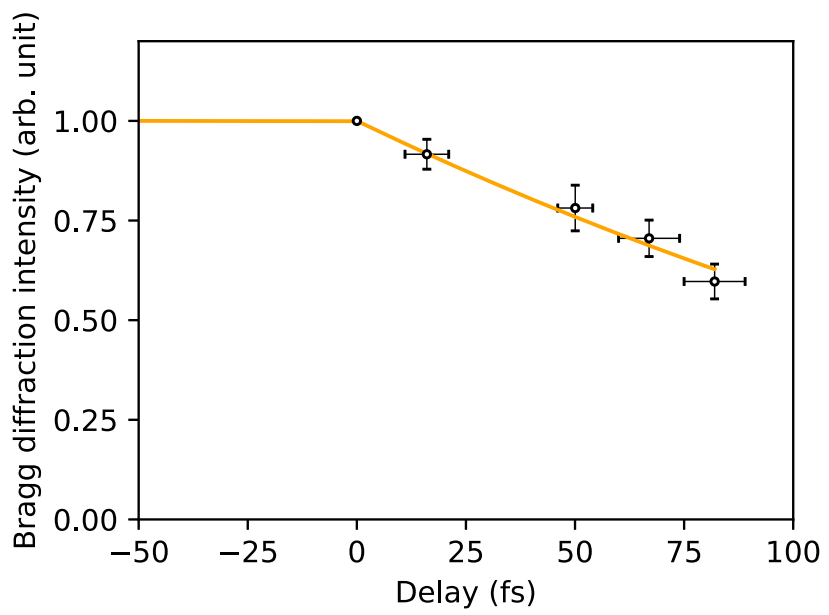

FIG. 8. Delay-dependent Bragg diffraction intensity of Xe clusters irradiated by intense $\mathrm{x}$-ray pulses. The data were reproduced from Ferguson et al. [27]. The diffraction intensity was fitted with the surface "melting" model (orange). kinetic energy because it does not explicitly depend on $\sigma_{q}^{\mathrm{KE}}$ [see Eq. (B5)].

\section{APPENDIX C: ANALYSIS OF THE X-RAY-X-RAY PUMP-PROBE DIFFRACTION DATA}

The experimental data are taken from Ferguson et al. [27]. In their work, atomic displacement $\Delta x$ was derived via $I / I_{0}=\exp \left(-q^{2} \Delta x^{2} / 3\right)$, where $q=2.928 \AA^{-1}$ is the momentum transfer of the $\{220\}_{\text {fcc }}$ peak, and $I / I_{0}$ is the delay-dependent Bragg diffraction intensity of the $\{220\}_{\text {fcc }}$ peak normalized by the initial intensity. We first reproduce the delay-dependent diffraction intensity $I / I_{0}$ from $\Delta x$ given in Fig. 2 of Ferguson et al. [27]. The diffraction intensity is fitted with the surface "melting" model expressed by Eqs. (6) and (7). Here, we employ $R=$ $35 \mathrm{~nm}$ [27] and $B=0$. The fitting result is displayed in Fig. 8 . The fitting gives a value of $(6.2 \pm 0.6) \times 10^{4} \mathrm{~m} / \mathrm{s}$ for the propagation speed $v_{\text {prop }}$.

[1] D. Strickland and G. Mourou, Compression of Amplified Chirped Optical Pulses, Opt. Commun. 56, 219 (1985).

[2] F. Krausz and M. Ivanov, Attosecond Physics, Rev. Mod. Phys. 81, 163 (2009).

[3] K. K. Ostrikov, F. Beg, and A. Ng, Colloquium: Nanoplasmas Generated by Intense Radiation, Rev. Mod. Phys. 88, 011001 (2016).

[4] A. H. Zewail, Femtochemistry: Atomic-Scale Dynamics of the Chemical Bond, J. Phys. Chem. A 104, 5660 (2000).

[5] D. M. Jonas, Two-Dimensional Femtosecond Spectroscopy, Annu. Rev. Phys. Chem. 54, 425 (2003).

[6] M. Nisoli, P. Decleva, F. Calegari, A. Palacios, and F. Martín, Attosecond Electron Dynamics in Molecules, Chem. Rev. 117, 10760 (2017).

[7] W. Steen and J. Mazumder, Laser Material Processing (Springer-Verlag, London, 2010).

[8] D. Bäuerle, Laser Processing and Chemistry (Springer, Berlin, Heidelberg, 2011).

[9] W. Ackermann et al., Operation of a Free-Electron Laser from the Extreme Ultraviolet to the Water Window, Nat. Photonics 1, 336 (2007).

[10] P. Emma et al., First Lasing and Operation of an AngstromWavelength Free-Electron Laser, Nat. Photonics 4, 641 (2010).

[11] N. D. Loh et al., Fractal Morphology, Imaging and Mass Spectrometry of Single Aerosol Particles in Flight, Nature (London) 486, 513 (2012).

[12] L. Redecke et al., Natively Inhibited Trypanosoma Brucei Cathepsin B Structure Determined by Using an X-Ray Laser, Science 339, 227 (2013).

[13] A. Doerr, Diffraction before Destruction, Nat. Methods 8, 283 (2011).

[14] T. Ditmire, J. W. Tisch, E. Springate, M. B. Mason, N. Hay, R. A. Smith, J. Marangos, and M. H. Hutchinson, HighEnergy Ions Produced in Explosions of Superheated Atomic Clusters, Nature (London) 386, 54 (1997). 
[15] M. Lezius, S. Dobosz, D. Normand, and M. Schmidt, Explosion Dynamics of Rare Gas Clusters in Strong Laser Fields, Phys. Rev. Lett. 80, 261 (1998).

[16] T. Fennel, T. Döppner, J. Passig, C. Schaal, J. Tiggesbäumker, and K.-H. Meiwes-Broer, Plasmon-Enhanced Electron Acceleration in Intense Laser Metal-Cluster Interactions, Phys. Rev. Lett. 98, 143401 (2007).

[17] A. McPherson, B. D. Thompson, A. B. Borisov, K. Boyer, and C. K. Rhodes, Multiphoton-Induced X-Ray Emission at 4-5 keV from Xe Atoms with Multiple Core Vacancies, Nature (London) 370, 631 (1994).

[18] W. A. Schroeder, F. G. Omenetto, A. B. Borisov, J. W. Longworth, A. McPherson, C. Jordan, K. Boyer, K. Kondo, and C. K. Rhodes, Pump Laser Wavelength-Dependent Control of the Efficiency of Kilovolt X-Ray Emission from Atomic Clusters, J. Phys. B 31, 5031 (1998).

[19] C. Bostedt, E. Eremina, D. Rupp, M. Adolph, H. Thomas, M. Hoener, A. R. B. de Castro, J. Tiggesbäumker, K.-H. Meiwes-Broer, T. Laarmann, H. Wabnitz, E. Plönjes, R. Treusch, J. R. Schneider, and T. Möller, Ultrafast X-Ray Scattering of Xenon Nanoparticles: Imaging Transient States of Matter, Phys. Rev. Lett. 108, 093401 (2012).

[20] T. Gorkhover et al., Nanoplasma Dynamics of Single Large Xenon Clusters Irradiated with Superintense X-Ray Pulses from the Linac Coherent Light Source Free-Electron Laser, Phys. Rev. Lett. 108, 245005 (2012).

[21] T. Gorkhover et al., Femtosecond and Nanometre Visualization of Structural Dynamics in Superheated Nanoparticles, Nat. Photonics 10, 93 (2016).

[22] T. Nishiyama et al., Ultrafast Structural Dynamics of Nanoparticles in Intense Laser Fields, Phys. Rev. Lett. 123, 123201 (2019).

[23] P. Mora, Plasma Expansion into a Vacuum, Phys. Rev. Lett. 90, 185002 (2003).

[24] Y. V. Medvedev, Expansion of a Finite Plasma into a Vacuum, Plasma Phys. Controlled Fusion 47, 1031 (2005).

[25] S. J. Gitomer, R. D. Jones, F. Begay, A. W. Ehler, J. F. Kephart, and R. Kristal, Fast Ions and Hot Electrons in the Laser-Plasma Interaction, Phys. Fluids 29, 2679 (1986).

[26] T. Fennel, K. H. Meiwes-Broer, J. Tiggesbäumker, P. G. Reinhard, P. M. Dinh, and E. Suraud, Laser-Driven Nonlinear Cluster Dynamics, Rev. Mod. Phys. 82, 1793 (2010).

[27] K. R. Ferguson, M. Bucher, T. Gorkhover, S. Boutet, H. Fukuzawa, J. E. Koglin, Y. Kumagai, A. Lutman, A. Marinelli, M. Messerschmidt, K. Nagaya, J. Turner, K. Ueda, G. J. Williams, P. H. Bucksbaum, and C. Bostedt, Transient Lattice Contraction in the Solid-to-Plasma Transition, Sci. Adv. 2, e1500837 (2016).

[28] K. Tono, T. Togashi, Y. Inubushi, T. Sato, T. Katayama, K. Ogawa, H. Ohashi, H. Kimura, S. Takahashi, K. Takeshita, H. Tomizawa, S. Goto, T. Ishikawa, and M. Yabashi, Beamline, Experimental Stations and Photon Beam Diagnostics for the Hard X-Ray Free Electron Laser of SACLA, New J. Phys. 15, 083035 (2013).

[29] T. Ishikawa et al., A Compact X-Ray Free-Electron Laser Emitting in the Sub-Ångström Region, Nat. Photonics 6, 540 (2012).

[30] H. Fukuzawa, K. Nagaya, and K. Ueda, Advances in Instrumentation for Gas-Phase Spectroscopy and Diffraction with Short-Wavelength Free Electron Lasers,
Nucl. Instrum. Methods Phys. Res., Sect. A 907, 116 (2018).

[31] Y. Inubushi, K. Tono, T. Togashi, T. Sato, T. Hatsui, T. Kameshima, K. Togawa, T. Hara, T. Tanaka, H. Tanaka, T. Ishikawa, and M. Yabashi, Determination of the Pulse Duration of an X-Ray Free Electron Laser Using Highly Resolved Single-Shot Spectra, Phys. Rev. Lett. 109, 144801 (2012).

[32] T. Katayama, T. Hirano, Y. Morioka, Y. Sano, T. Osaka, S. Owada, T. Togashi, and M. Yabashi, X-Ray Optics for Advanced Ultrafast Pump-Probe X-Ray Experiments at SACLA, J. Synchrotron Radiat. 26, 333 (2019).

[33] O. F. Hagena, Cluster Ion Sources (Invited), Rev. Sci. Instrum. 63, 2374 (1992).

[34] Y. Kumagai et al., Following the Birth of a Nanoplasma Produced by an Ultrashort Hard-X-Ray Laser in Xenon Clusters, Phys. Rev. X 8, 031034 (2018).

[35] T. Katayama, S. Owada, T. Togashi, K. Ogawa, P. Karvinen, I. Vartiainen, A. Eronen, C. David, T. Sato, K. Nakajima, Y. Joti, H. Yumoto, H. Ohashi, and M. Yabashi, A Beam Branching Method for Timing and Spectral Characterization of Hard X-Ray Free-Electron Lasers, Struct. Dyn. 3, 034301 (2016).

[36] T. Kameshima, S. Ono, T. Kudo, K. Ozaki, Y. Kirihara, K. Kobayashi, Y. Inubushi, M. Yabashi, T. Horigome, A. Holland, K. Holland, D. Burt, H. Murao, and T. Hatsui, Development of an X-Ray Pixel Detector with MultiPort Charge-Coupled Device for X-Ray Free-Electron Laser Experiments, Rev. Sci. Instrum. 85, 033110 (2014).

[37] G. Bradski, The OpenCV Library, Dr Dobbs J. Software Tools 25, 120 (2000).

[38] A. Niozu et al., Characterizing Crystalline Defects in Single Nanoparticles from Angular Correlations of Single-Shot Diffracted X-Rays, IUCrJ 7, 276 (2020).

[39] J. W. Tisch, N. Hay, K. J. Mendham, E. Springate, D. R. Symes, A. J. Comley, M. B. Mason, E. T. Gumbrell, T. Ditmire, R. A. Smith, J. P. Marangos, and M. H. Hutchinson, Interaction of Intense Laser Pulses with Atomic Clusters: Measurements of Ion Emission, Simulations and Applications, Nucl. Instrum. Methods Phys. Res., Sect. B 205, 310 (2003).

[40] D. Rupp, M. Adolph, T. Gorkhover, S. Schorb, D. Wolter, R. Hartmann, N. Kimmel, C. Reich, T. Feigl, A. R. B. de Castro, R. Treusch, L. Strüder, T. Möller, and C. Bostedt, Identification of Twinned Gas Phase Clusters by SingleShot Scattering with Intense Soft X-Ray Pulses, New J. Phys. 14, 055016 (2012).

[41] T. Nishiyama et al., Refinement for Single-Nanoparticle Structure Determination from Low-Quality Single-Shot Coherent Diffraction Data, IUCrJ 7, 10 (2020).

[42] C. Peltz, C. Varin, T. Brabec, and T. Fennel, Time-Resolved $X$-Ray Imaging of Anisotropic Nanoplasma Expansion, Phys. Rev. Lett. 113, 133401 (2014).

[43] T. Döppner, J. P. Müller, A. Przystawik, J. Tiggesbäumker, and K.-H. Meiwes-Broer, The Effect of Volumetric Weighting in the Interaction of Intense Laser Fields with Clusters, Eur. Phys. J. D 43, 261 (2007).

[44] R. F. Schmalz, New Self-Similar Solutions for the Unsteady One-Dimensional Expansion of a Gas into a Vacuum, Phys. Fluids 28, 2923 (1985). 
[45] S. P. Hau-Riege, R. A. London, and A. Szoke, Dynamics of Biological Molecules Irradiated by Short X-Ray Pulses, Phys. Rev. E 69, 051906 (2004).

[46] D. Rupp, M. Adolph, L. Flückiger, T. Gorkhover, J. P. Müller, M. Müller, M. Sauppe, D. Wolter, S. Schorb, R. Treusch, C. Bostedt, and T. Möller, Generation and Structure of Extremely Large Clusters in Pulsed Jets, J. Chem. Phys. 141, 044306 (2014).

[47] M. J. Bucher, Ultrafast Dynamics of Nanoparticles in Highly Intense X-Ray Pulses, Ph.D. thesis, Technische Universität Berlin (2017).

[48] D. Rupp, L. Flückiger, M. Adolph, T. Gorkhover, M. Krikunova, J. P. Müller, M. Müller, T. Oelze, Y.
Ovcharenko, B. Röben, M. Sauppe, S. Schorb, D. Wolter, R. Mitzner, M. Wöstmann, S. Roling, M. Harmand, R. Treusch, M. Arbeiter, T. Fennel, C. Bostedt, and T. Möller, Recombination-Enhanced Surface Expansion of Clusters in Intense Soft X-Ray Laser Pulses, Phys. Rev. Lett. 117, 153401 (2016).

[49] M. Lebeault, J. Viallon, J. Chevaleyre, C. Ellert, D. Normand, M. Schmidt, O. Sublemontier, C. Guet, and B. Huber, Resonant Coupling of Small Size-Controlled Lead Clusters with an Intense Laser Field, Eur. Phys. J. D 20, 233 (2002). 